\title{
RETRIEVING STEREO IMAGE USING VISUAL WORD PAIRS WITH CONVOLUTION NEURAL NETWORK
}

\author{
Rachakonda Swetha \\ PG Student, Department of ECE, \\ Kakatiya Institute of technology and science, Warangal \\ rachakondadhana4@gmail.com \\ Ganta Raghotham Reddy \\ Professor, Department of ECE, \\ Kakatiya Institute of technology and science, Warangal \\ grrece9@gmail.com
}

\begin{abstract}
The exponential increase and omnipresent access to visual data on the Internet contributed to the growth of image search or recovery science. Ignoring the visual content as a ranking indicator can result in inconsistencies between the words and the visual content with the selection technique. The failure of current methods and characteristics of HrosSIs therefore does not provide a reasonable retrieval of images. In addition, nothing of the recent remote sensing image retrieval techniques takes the basic features of highresolution optical satellite stereo images (HrosSIs) such as the display number and the viewing angles into account. The article offers a specific structure to leverage the unique features of HrosSIs data in such a way that HrosSI's information is collected efficiently and accurately. The recovery of HrosSI is achieved through the similitude among the structures attained from DSMs and orthographs, together mined from the HrosSIs. Moreover, the value of pinnacle evidence stood examined for HrosSI recovery. For process authentication spending the ISPRS stereo test dataset, a prototype framework has been developed and implemented. Investigational results demonstration that the methods projected for HrosSI recovery are successful. The proposed system is successful and tailored to stereo images conveyed across space but may also be sufficient for stereo images transmitted by air.
\end{abstract}

Keywords: Image Retrieval; Visually word pairs; Stereo Images and Convolutional Neural Network (CNN).

\section{Introduction}

Since digital devices are increasingly common and embedded in cameras and fast Internet technology is growing, millions are planned to share web images and navigate them. Light is shone on many new applications focused on image search through the all-round exposure to both physical and Internet images. Image search aims at efficiently retrieving correct visual materials from a broad visual corpus for a textual or visual query. Although Image searches have been widely discussed since the start of the 1990s [1]. Thanks to attention to the challenges of scalability and emerging technologies they nevertheless receive tremendous interest from the multimedia plus computer vision groups in the last epoch. Normal image search engines usually index montage visual statistics based on neighboring web images, such for instance titles and tags. Due to the incompatibility of text information with visual content, content-based retrieval of images (CBIR) would be favored and shown in recent years to be quite relevant. The number of images generated is increasingly growing with more digital applications. In order to retrieve relevant images, enormous image repositories must be searched; this is specified as CBIR systems. A physical quest of these image databases remains not only widespread but also not fruitful, so different approaches to improved image retrieval have been proposed. CBIR is therefore regarded as an intense area of research [1-3] and is therefore difficult to identify sights correctly after a large amount of digital images. The text-based demand methods commonly used are not as dependable plus accurate as practical approaches. The different embryonic topographies like tint, contour, construction, surface, etc. can be used for the identification of the images previously stowed in a database plus a user probe [4-8]. 
Nevertheless, the hierarchical nature of HrosSI data makes it hard to obtain similar characteristics from different HrosSIs for HrosSI content recovery. HrosSIs are acquired under different remote sensors and differ widely, for example, in stereo acquisition, visual numbers, observing angles, conjunction angles, base to height ratio, soil distance model (GSD), RSD and metadata construction. In current content-based image recovery methods certain specific individualities of HrosSIs statistics are not demoralized; thus, their capacity for extraction of features and recovery is unknown. To ensure an effective and reliable HrosSI retrieval, the value of height evidence needs to be examined. It is easy to obtain height data from stereo mined DSMs, a principal merchandise form generated from HrosSI data. Though, in current image retrieval methods height info is not occupied hooked on excuse. Height data canister is useful in distinguishing between forms of land cover which are difficult to differentiate from flat features alone. For example, on a plain land and in mountains, forest terrestrial has similar texture characteristics, but dramatically differs in height. High knowledge may thus be useful for HrosSI content-based recovery.

This paper proposes a general HrosSI content recovery structure that takes into consideration the height data. In particular we concentrate on: 1) topographies can remain uniformly extracted from numerous HrosSIs attained from various remote sensors underneath a variety of gaining conditions and 2) if height information is useful aimed at HrosSIs recall.

\section{Literature Review}

Jegou et al. claimed that visual terms have some drawbacks with the closest neighbor matching techniques. Many true matches can be missed for a large visual dictionary and a small dictionary can contain several false matches. Hamming embedding solves the problem by computing for a descriptor a binary signature and integrating the binary signature distance in the nearest neighboring location. The introduction of a poor geometric consistency test often improves the image classification system of previous approaches. This is low-cost and can be extended to all images, in which earlier geometric checklists are only a few hundred images in large datasets.

A very similar work was put forward by Knopp et al. Their approach is by matching an urban base of streetside images to identify a position in a query image. We know that many common items such as trees, road signs, vehicles, etc. can be used for misunderstanding. Nevertheless, the advantages of geo-tags in the images are used to eliminate misleading objects.

Visual synonyms were described by Gavves and Snoek in 2010. We use a similar concept as well as classify the images according to the similarity of the question training function only to further process the top graded images for geometrical checking. By estimating a homographic matrix, geometric connectivity is calculated. The corresponding pair of words is a candidate for visual synonyms by two similar features mapped to separate visual terms but consistent in geometric location. Word pairs, which occur very rarely and are known as visual synonyms for the rest of the candidates. The top images with the visual synonyms are the corresponding images of the retrieval process.

In addition, Du et al. proposed the combination of RGB and 3D model depth data. They divide into local alignment and global alignment the recording process. In addition, this is similar since local alignment processes frame-to-frame tracking and global alignment uses loop closing information in order to refine the frame poses to conform to a world-wide frame of reference. The concept of key frames for the loop closure is used here. The difference between this approach and this is that Du et al. also implemented an error handling user input program. The program will notify the user whether the registration error happens and the user will re-start. The user can test it via visualization when a loop closure has been detected.

The program will notify the user whether the registration error happens and the user will re-start. The user can test it via visualization when a loop closure has been detected.

\section{Materials And Methods}

For various HrosSIs obtained from various remote sensors under different acquisition conditions, a new generic frame is proposed. The HrosSI recall is performed with the similarity of the two primary HrosSI stereo generated features derived from DSMs and ortho images.

The differentiation between HrosSIs with diverse appearances takes many steps. The first process is to produce these dual main stereo harvests for specific HrosSIs, since the similarity between HroSI is performed by a similarity between stereo extracted ortho-images and DSMs. Secondly, the complex and very rich visual content of large-size images makes it laborious to type similar judgments on the appearance; consequently, these big HrosSIs plus stereo-products necessity remain broken down into many retrieval blocks. When matching these smaller items, a query template is helpful. 


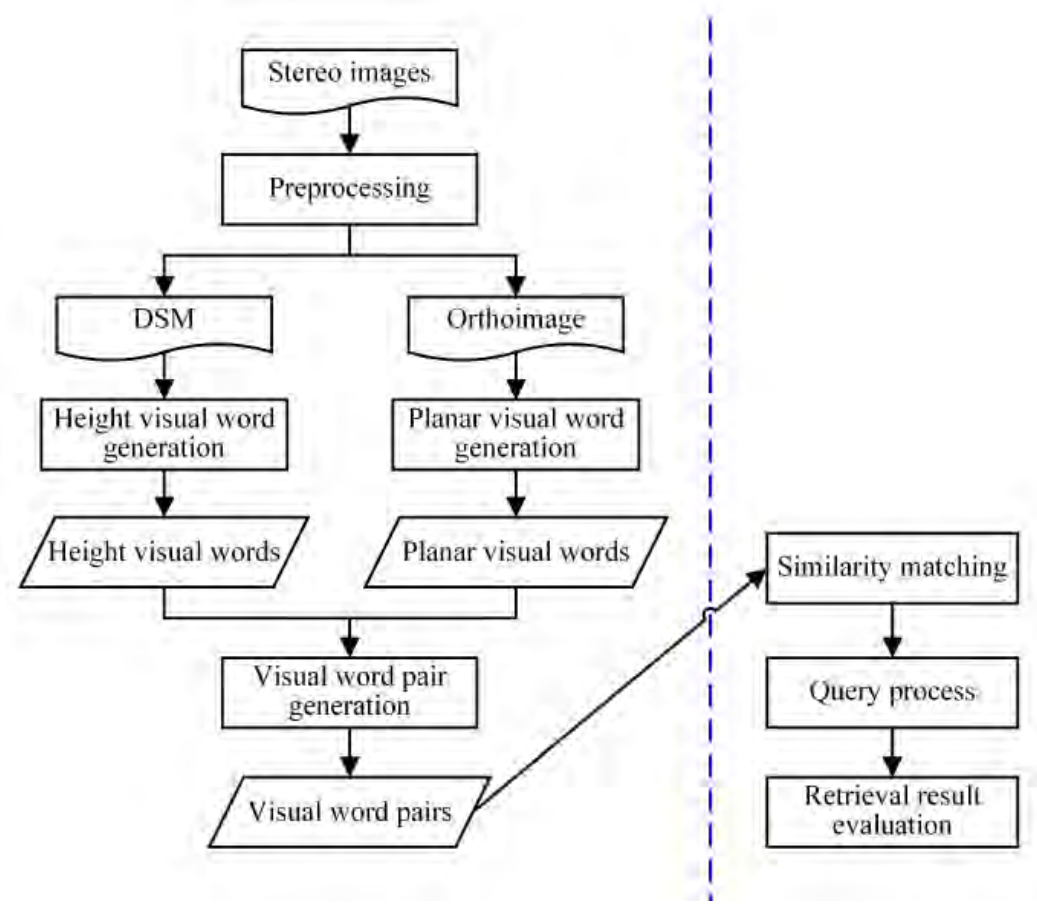

Fig.1. Flow chart for Stereo image retrieval.

To achieve similarity between HrosSIs, stereo products are produced. Since stereo product characteristics that affect the similarity of HrosSIs, certain steps must be taken to reduce the effects of these characteristics. Digital surface models with a single GSD are produced using the same matching algorithm from various HrosSIs. GSD and image information still differ in DSMs created with various HrosSIs. Accordingly, DSMs will then be resampled and filtered with a low pass. In addition, orthorexes of the near-nadir image between the HrosSI groups are created with the help of stereo extracted DSMs. Orthodontics created from different HrosSIs quiet differ in the GSD, radiometric firmness, image strength, and image information, which is why orthoic images must be resampled, normalized radiometric resolution, equalized histograms and filtering of the smaller passes. HrosSIs and large-sized stereo items are divided into several recall blocks, as shown in Figure. 2.

Increasing image is decomposed into equal size non-overlapping blocks using the same setting in orthography, HrosSIs and DSM.

By means of a block-oriented image decomposition process, HrosSIs are decomposed to the same size, not overlapping HrosSI block groups. These classes of HrosSI, which are regarded as smaller HrosSIs, are used for the recovery of HrosSIs

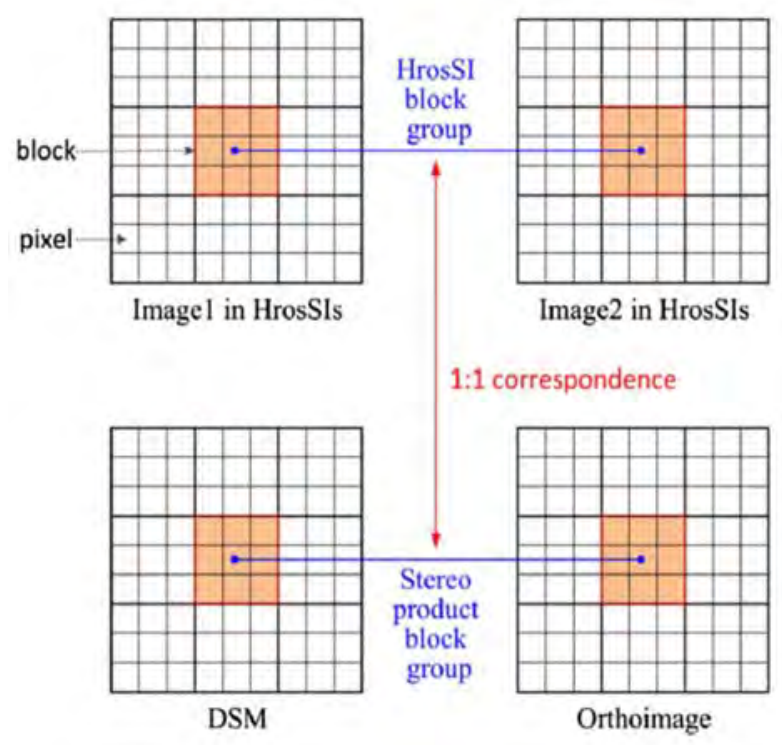

Fig.2. HrosSIs and stereo products decomposition. 
With their stereo products, the same decomposition environment is used to generate multiple stereo block groups. In order to ensure that the function derived from a HrosSI block group is considered a function of a specific HrosSIlump group, the one-to- one communication is constructed among a HrosSI block group plus their consistent stereo wedge group.

\subsection{Graphic word pair generation}

A spot pair which involves of digital surface model cover and orthoimage patch is known as coupled patch pair. Pinnacle and planar code words remain arranged from smaller towards bigger.

$$
w p_{(i, j)}=(i, j) 1 \leq i \leq k, 1 \leq j \leq k
$$

Here $w p_{(i, j)}$ is known as pictorial announcement pair for the coupled patch pair and $\mathrm{i}, \mathrm{j}$ are sequential order amounts of planar and stature code words.

Resemblance identical between two stereophonic pairs or trios is performed through means of likeness identical amid their pictorial word sets.

$$
\operatorname{similarity}\left(\mathrm{im}_{1}, \mathrm{im}_{2}\right)=\sum_{i=1}^{k} \sum_{j=1}^{k} \operatorname{sim}\left(w p_{i, j}^{1}, w p_{i, j}^{2}\right)
$$

\subsection{Evaluation of retrieval result}

The correctness of the reclamation technique is calculated primarily built on ranking listing that retorts to a question with reclamation system

$$
\text { Precision }=\frac{T P}{T P+F P}
$$

Here, TP is the amount of true positives, FN is the amount of false negatives and FP is the amount of false positives The retrieval effectively remains measured with the time from the question to the retrieval outcomes return to users.

\subsection{Convolutional neural network}

A CNN is a neural community that has one or greater convolutional layers and are used by and large for photo processing. Convolutional is gr-eater friendly due to the fact it reduces the variety of parameters. The most frequent use for $\mathrm{CNN}$ image classification, for instance figuring out satellite images. Convolutional Neural Network (CNN) is a Deep Learning algorithm which can take in an enter image, assign significance such as learnable weights and biases to a range of aspect in the photograph and be capable to differentiate one from another.

\section{layer $\mathrm{m}-\mathrm{I}$}

\section{hidden layer $\mathrm{m}$}

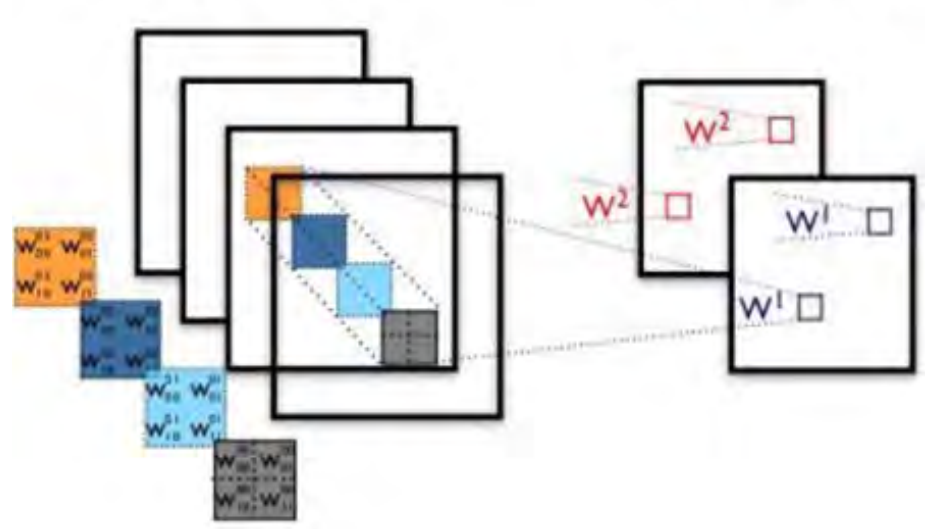

Fig.3. Convolutional Neural Network hidden layers

\subsection{Analysis of efficiency calculation}

Figure. 4 shows the image retrieval efficiency which depends upon the feature extraction of our method and CNN. For all those methods we have taken a set of 20 images. The extraction of feature is an offline process, the feature extraction is time consumed process and image retrieval method is fast here and we can see the all output images at a time on display. We are using $\mathrm{CNN}$ in our method so here it initializes a network and then train a network so that it can a little bit time in order to evaluate each and every point and gives us best precision. In accumulation, this technique taken abundant high recovery efficiency than that of previous methods. 


\section{Simulation Results And Discussions}

The performance of the proposed method as well as existing method is evaluated with precision parameter. The precision parameter value within the range of 0 to 1 , higher precision value leads the superior retrieval results and lower precision value leads the very low retrieval results of both the methods. The simulation results of the proposed CNN method is shown in figures 3 and 4 respectively. Similarly, figure 4 depicts the efficiency calculation using both the methods. The table 1 shows the comparison table for both existing and proposed retrieval methods in terms of precision. The table 1 clearly shows the proposed CNN retrieval method got the higher value of the precision, it shows the utmost performance of the proposed method over the existing DSM and DSM plus ORTHO methods. Existing DSM and DSM plus Ortho retrieval methods got the moderate values of the precision, which shows the moderate accuracy and precision values over the proposed method. The retrieval data set is taken from the internet freely available to download.

Our method used set of 20 images in the data base and having the higher precision because of using convolutional neural network.

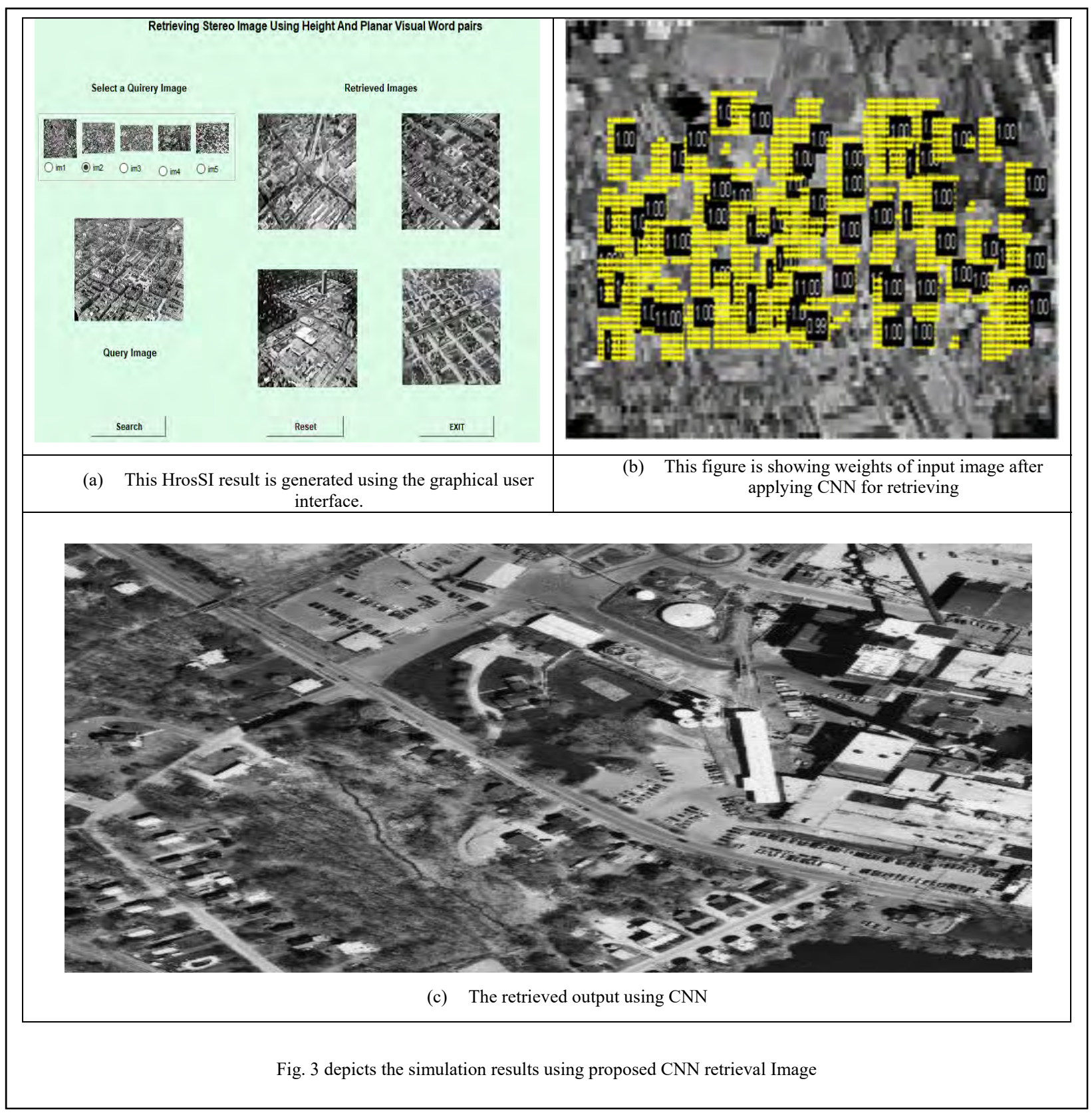




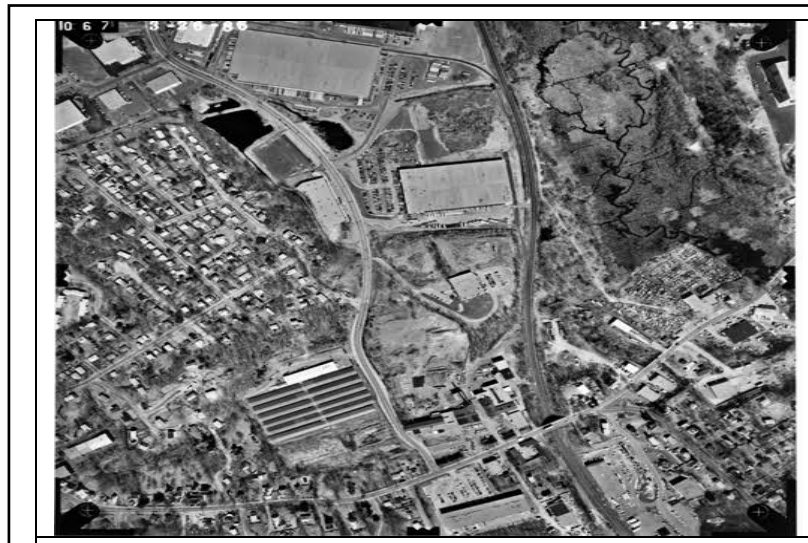

(a) This HrosSI result is generated using the graphical user interface.

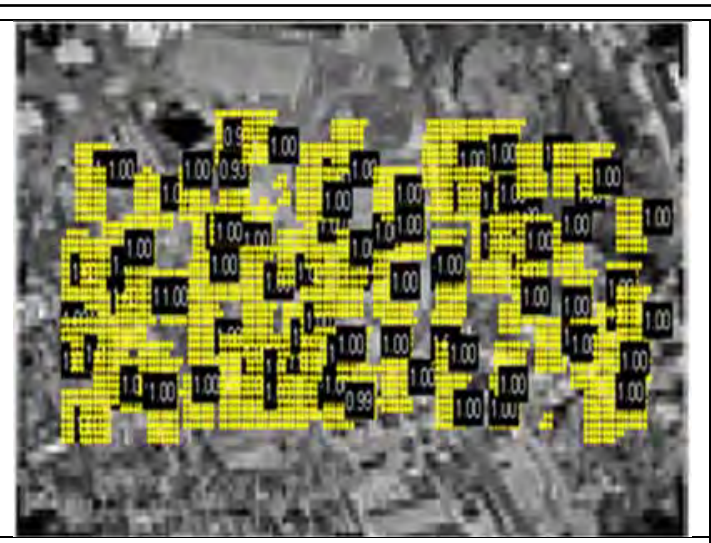

(b) This figure is showing weights of input image after applying CNN for retrieving

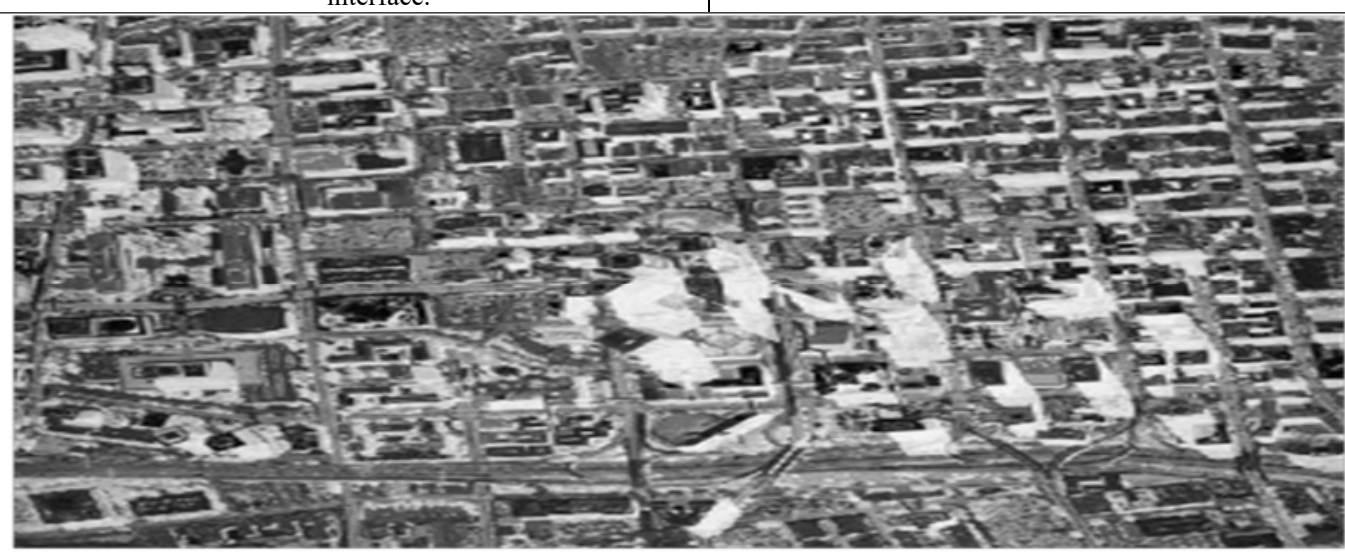

(c) The retrieved output using $\mathrm{CNN}$

Fig.4. Depicts the simulation results using proposed CNN retrieval Image

Table1: This table shows the comparison between precisions of existing methods and our method.

\begin{tabular}{|c|c|c|c|}
\hline S.NO & DSM Precision & DSM+ORTHO Precision & CNN Precision \\
\hline 1 & 0.45 & 0.72 & 0.88 \\
\hline 2 & 0.66 & 0.84 & 0.95 \\
\hline 3 & 0.77 & 0.78 & 0.96 \\
\hline 4 & 0.85 & 0.88 & 0.98 \\
\hline
\end{tabular}

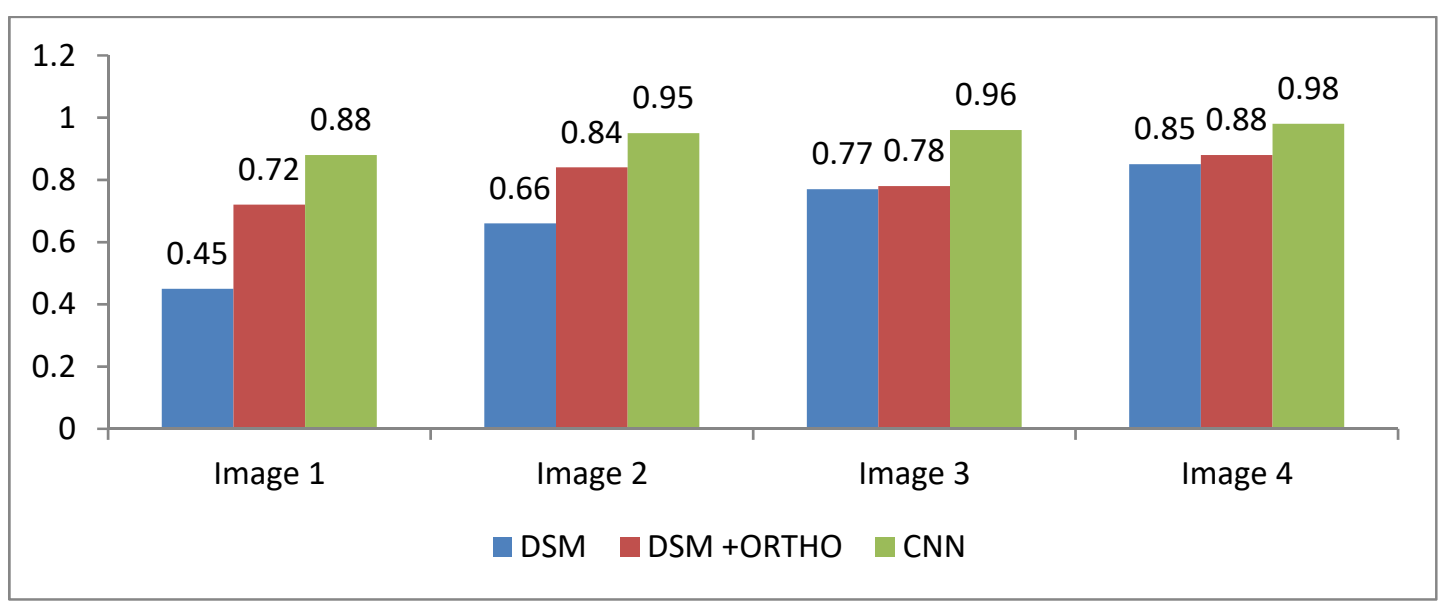

Fig.4.depicts the efficiency calculation using proposed and existing retrieval methods. Here y-axis is taken as precision and the x-axis are Images. 


\section{Conclusion}

This paper presents a new standardized structure for HrosSI material recovery and discusses the relevance of highlevel knowledge for HrosSI recovery. HrosSI is done via a similarity of features derived from stereo-extracted orthoimages and DSMs. Exploratory outcomes show that the techniques the HrosSI recuperation suggestions succeed. Stereophonic goods made after different HrosSIs must be of the alike radiometric resolution and GSD in order to achieve the most accurate HrosSI reproduction. High info is ineffectual then unsteady aimed at HrosSI retrieval by itself, but a union of flat information and high information is stable and efficient for HrosSI recovery.

\section{References}

[1] N. Shrivastava and V. Tyagi, (2014): Content based image retrieval based on relative locations of multiple regions of interest using selective regions matching, Information Sciences, vol. 259, pp. 212-224.

[2] N. S. Mansoori, M. Nejati, P. Razzaghi, and S. Samavi, (2013): Bag of visual words approach for image retrieval using color information," in Proceedings of the 2013 21st Iranian Conference on Electrical Engineering, ICEE 2013, Mashhad, Iran.

[3] G.-H. Liu and J.-Y. Yang, (2013): Content-based image retrieval using color difference histogram, Pattern Recognition, vol. 46, no. 1, pp. 188-198.

[4] G.-L. Shen and X.-J. Wu, (2013): Content based image retrieval by combining color texture and CENTRIST," in Proceedings of the Constantinides International Workshop on Signal Processing (CIWSP '13), pp. 1-4.

[5] A. Talib, M. Mahmuddin, H. Husni, and L. E. George, (2013): A weighted dominant color descriptor for content-based image retrieval, Journal of Visual Communication and Image Representation, vol. 24, no. 3, pp. 345-360

[6] B.-M.Chang, H.-H. Tsai, andW.-L.Chou, (2013): Using visual features to design a content-based image retrieval method optimized by particle swarm optimization algorithm, Engineering Applications of Artificial Intelligence, vol. 26, no. 10, pp. 2372-2382.

[7] G.-H. Liu, Z.-Y. Li, L. Zhang, and Y. Xu, (2011): Image retrieval based on micro-structure descriptor, Pattern Recognition, vol. 44, no. 9, pp. 2123-2133.

[8] M. E. Elalami, (2014): A new matching strategy for content based image retrieval system, Applied Soft Computing, vol. 14, pp. 407418.

[9] G.W. Jiji and P. J. Durairaj, (2015): Content-based image retrieval techniques for the analysis of dermatological lesions using particle swarm optimization technique, Applied Soft Computing, vol. 30, pp. 650-662.

[10] X.-Y. Wang, Y.-J. Yu, and H.-Y. Yang, (2011): An effective image retrieval scheme using color, texture and shape features, Computer Standards \& Interfaces, vol. 33, no. 1, pp. 59-68. 